\title{
Tratamento cirúrgico da ascite quilosa
}

\author{
Surgical treatment of chylous ascites \\ Claudia Stein Gomes ${ }^{1}$, Gustavo Ioshio Handa ${ }^{1}$, Fabio Porto Silveira ${ }^{2}$, Vinicius Zendrini Buzingnani ${ }^{1}$, \\ Fernanda Macedo Binati ${ }^{1}$, Elizana Stella Lopes Rasera ${ }^{1}$
}

\section{Resumo}

A ascite quilosa é uma complicação rara após procedimentos cirúrgicos e trauma abdominal, apresentando elevada morbidade e difícil manejo. Nos casos refratários ao tratamento clínico habitual, o tratamento cirúrgico se impõe, apesar da baixa taxa de sucesso. Dois casos são apresentados: o primeiro paciente foi vítima de trauma abdominal contuso e o segundo foi submetido a hernioplastia hiatal a Nissen videolaparoscópica, ambos evoluindo com ascite quilosa que necessitou de tratamento cirúrgico através da ligadura do ducto torácico, junto aos pilares diafragmáticos. Os pacientes evoluíram com melhora clínica e ausência de ascite após 24 meses de seguimento.

Palavras-chave: Ascite quilosa, doenças linfáticas, laparoscopia.

\section{Introdução}

Ascite quilosa é uma rara complicação após procedimentos cirúrgicos abdominais e após trauma toracoabdominal. O tratamento clínico baseado em dieta rica em proteínas e baixo teor de gordura, nutrição parenteral, administração de somatostatina ou octreotide tem sido o tratamento inicial de eleição. Porém, nos casos em que a ascite é refratária a essas opções terapêuticas, o tratamento cirúrgico se impõe, apesar de não haver consenso na literatura sobre a melhor forma de realizá-lo e em qual momento. Os casos descritos mostram pacientes com ascite quilosa pós-operatória e pós-traumática tratada cirurgicamente com sucesso através de ligadura e esclerose do ducto torácico ao nível dos pilares diafragmáticos.

\begin{abstract}
Chylous ascites is a rare complication after abdominal procedures and blunt abdominal trauma, associated with high morbidity and difficult management. When clinical treatment fails, surgical intervention is necessary, despite the limited success rate. Two cases are reported: the first patient had a blunt abdominal trauma and the second patient underwent laparoscopic Nissen fundoplication; both developed chylous ascites and required surgical treatment with suture repair of the thoracic duct, close to the diaphragm. There was clinical improvement and ascites was absent at a 24-month follow-up.
\end{abstract}

Keywords: Chylous ascites, lymphatic diseases, laparoscopy.

\section{Relato dos casos}

\section{Caso 1}

Paciente do sexo feminino, 36 anos, vítima de colisão autoanteparo, apresentou trauma toracoabdominal, sendo atendida no pronto socorro de sua cidade, com quadro de dor torácica e dispneia leve. Fez radiografia de tórax, a qual não evidenciou alterações, e recebeu alta em seguida. Seis meses após o acidente, apresentava dispneia e dor suprapúbica, e foi observado líquido no fundo de saco de Douglas em ecografia pélvica, sendo então investigada com colonoscopia e endoscopia digestiva alta, sem alterações significativas que justificassem a ascite. Radiografia de tórax evidenciou derrame pleural bilateral, e a paciente foi submetida a toracocentese que evidenciou líquido de aspecto leitoso. Foi encaminhada para um serviço maior, sendo vastamente investigada para afastar causas neoplá-

1. Serviço de Angiologia e Cirurgia Vascular, Santa Casa de Misericórdia de Curitiba, Curitiba, PR.

2. Serviço de Cirurgia Geral, Santa Casa de Misericórdia de Curitiba, Curitiba, PR.

Apresentado no Congresso Brasileiro de Angiologia e Cirurgia Vascular em 2007.

Não foram declarados conflitos de interesse associados à publicação deste artigo.

Artigo submetido em 02.07.08, aceito em 29.12.08.

J Vasc Bras. 2009;8(2):192-197.

Copyright (C 2009 by Sociedade Brasileira de Angiologia e de Cirurgia Vascular 
sicas (biópsia hepática, biópsia de medula óssea, tomografia axial computadorizada de tórax e abdome), todas negativas para malignidade, firmando o diagnóstico de ascite e derrame pleural quiloso secundário ao trauma. Pela dificuldade do controle de débito da fistula $(>1.000 \mathrm{~mL} / \mathrm{dia})$, foi iniciada nutrição parenteral total associada a pleurodese química à direita. Paciente evoluiu com quadro séptico relacionado a infecção de cateter venoso central e síndrome da angústia respiratória, sendo encaminhada para UTI, onde permaneceu por 2 meses. Após melhora clínica e controle dos débitos, foi reintroduzida dieta via oral restritiva com suplementação nutricional parenteral, porém houve recidiva da ascite. Foram realizadas linfografia (Figura 1) e linfocintilografia (Figura 2), sem visualização do ducto torácico. A paciente retornou a jejum e nutrição parenteral total, sendo iniciada somatostatina, e evoluiu com melhora da ascite e reinício progressivo de dieta via oral restritiva até retirada da nutrição parenteral total com resolução aparente do caso e alta hospitalar.

Após 1 semana, paciente retorna com ascite volumosa e dor abdominal, sendo realizada drenagem de líquido quiloso (triglicerídeos $>1.000 \mathrm{mg} / \mathrm{dL}$ ), sem evidência de infecção (culturas negativas). Paciente foi então encaminhada para a Santa Casa de Misericórdia de Curitiba, em Curitiba (PR). Após estabilização clínica e investigação diagnóstica complementar através de tomografia de tórax e abdome (Figuras 3 e 4), a paciente foi submetida a laparotomia exploradora, com aspiração de $6 \mathrm{~L}$ de líquido ascítico quiloso, com acesso aos pilares diafragmáticos e ligadura dos coletores linfáticos nesse nível, com drenagem da cavidade abdominal com dreno de tubular. Paciente apresentou boa evolução pós-operatória, sem drenagem de secreção linfática pelo dreno e alta hospitalar no $10^{\circ}$ dia. Apresenta-se atualmente no $24^{\circ}$ mês pós-operatório, com estado nutricional normal e sem sinais de ascite nos exames (radiografia de tórax e ecografia abdominal) realizados semestralmente.

\section{Caso 2}

Paciente do sexo feminino, 50 anos, portadora de doença do refluxo gastroesofágico, apresentando hérnia hiatal volumosa, foi submetida a tratamento cirúrgico videolaparoscópico, sendo realizada redução do estômago para cavidade abdominal e fundoplicatura a Nissen. Apresentou boa evolução pós-operatória, recebendo alta no primeiro dia pós-operatório.

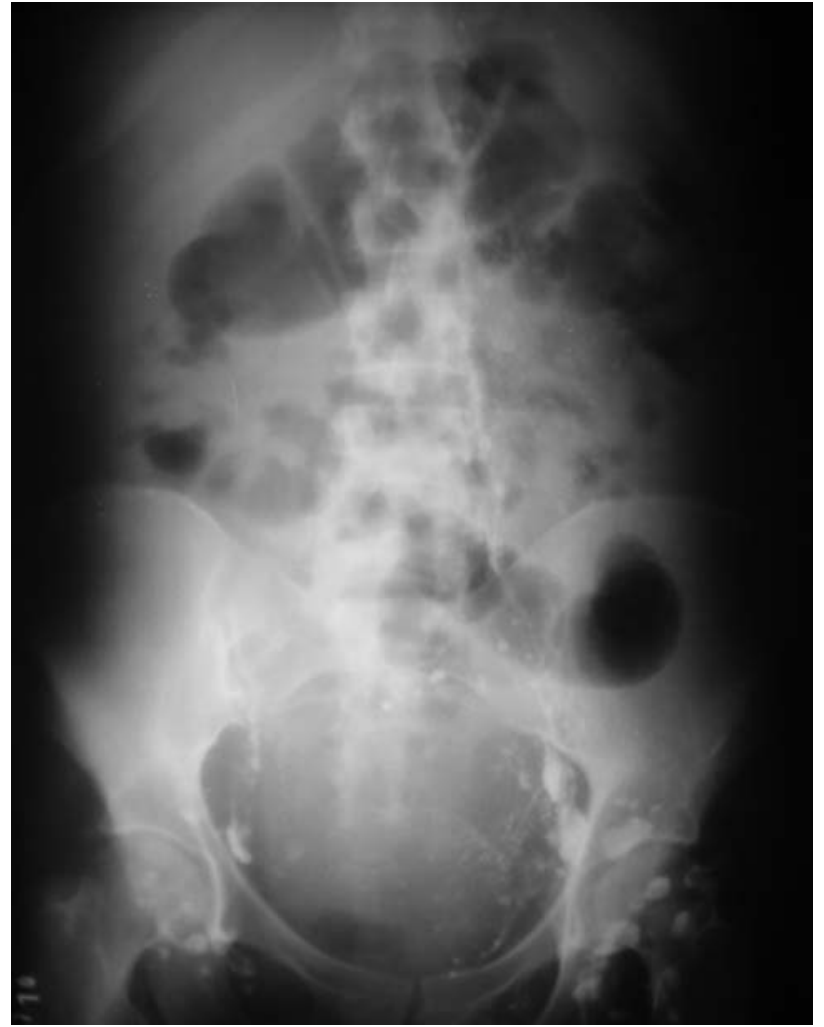

Figura 1 - Linfografia evidenciando progressão do contraste pelos vasos linfáticos sem extravazamento abdominal

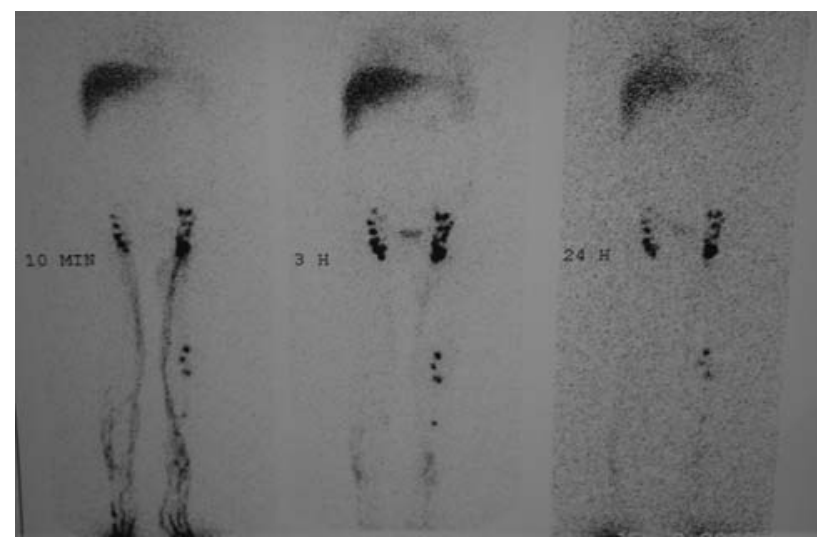

Figura 2 - Linfocintilografia sem evidência de lesão do ducto torácico

Trinta dias após a cirurgia, a paciente evoluiu com dor em hemitórax direito e dispneia leve, além de aumento do volume abdominal. Radiografia de tórax demonstrou derrame pleural volumoso à direita e ecografia de abdome total evidenciou líquido livre na cavidade peritoneal. Toracocentese demonstrou quilotórax. A paciente foi submetida a toracotomia direita e ligadura do ducto torácico ao nível da junção toracoabdominal e aspiração de ascite 


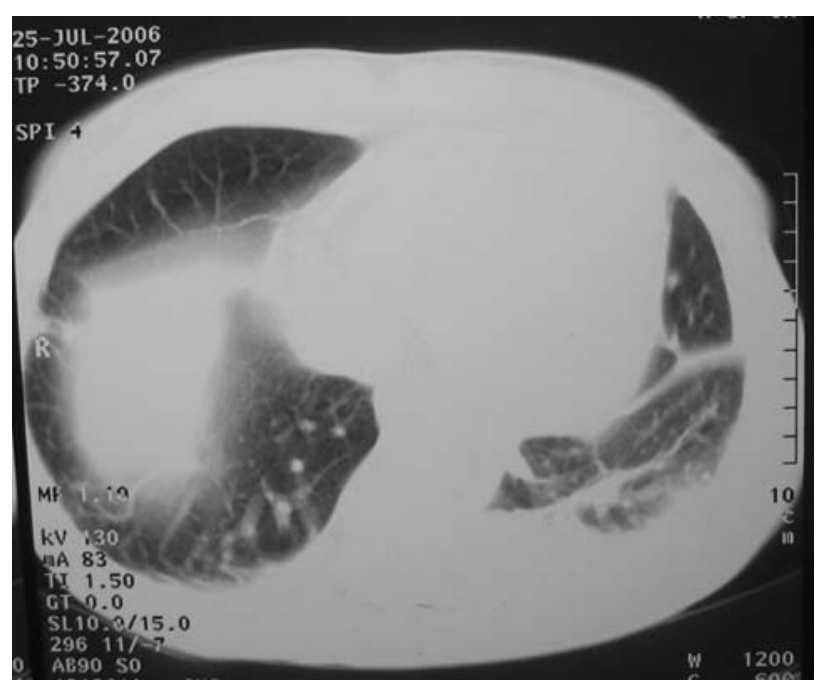

Figura 3 - Tomografia computadorizada de tórax evidenciando derrame pleural volumoso (quilotórax)



Figura 4 - Tomografia computadorizada de abdome evidenciando ascite quilosa

quilosa por via mediastinal, com drenagem de cerca de $4 \mathrm{~L}$ de líquido.

Evoluiu no pós-operatório com retorno da ascite quilosa, sendo submetida a nova videolaparoscopia para ligadura dos vasos linfáticos por via abdominal. No procedimento cirúrgico, foi realizada aspiração de $2 \mathrm{~L}$ de líquido ascítico quiloso, nova dissecção dos pilares diafragmáticos, sem detecção de ponto de vazamento. Foi também realizada passagem de óleo mineral por sonda nasogástrica, detectando-se vazamento de linfa junto aos pilares diafrag- máticos. Após dissecção local, não foi possível identificar o ducto, sendo realizada a esclerose de vasos linfáticos periaórticos com glicose $50 \%$, cessando a drenagem de secreção linfática. Realizou-se drenagem da cavidade abdominal com dreno de Blake, posicionado ao lado do pilar diafragmático.

Paciente permaneceu em nutrição parenteral total por 10 dias, sem drenagem de secreção quilosa pelo dreno. Iniciou, então, dieta sem gordura, apenas com triglicerídeos de cadeia média. Evoluiu após 20 dias de pós-operatório sem secreção quilosa pelo dreno, sendo retirado o mesmo e liberada dieta livre.

Paciente realizou ecografia de abdome total 30, 60 e 90 dias ao pós-operatório, sem evidência de ascite. Atualmente apresenta-se clinicamente bem, no vigésimo terceiro mês após a cirurgia.

\section{Discussão}

A ascite quilosa é um fluido peritoneal semelhante a leite e rico em triglicerídeos, com incidência estimada em 1 para 20.000 admissões em um grande hospital universitário ${ }^{1}$. Existem 3 mecanismos descritos para o desenvolvimento da ascite quilosa ${ }^{2}$ : obstrução do fluxo linfático por neoplasias malignas que causam vazamento por vasos linfáticos subserosos dilatados para a cavidade peritoneal; exsudação de linfa através das paredes de vasos retroperitoneais dilatados que drenam através de uma fístula para a cavidade peritoneal (linfangiectasia congênita); e obstrução do ducto torácico por trauma ou iatrogênico causando vazamento direto de quilo através de uma fístula linfoperitoneal.

As principais causas de ascite nos países ocidentais são neoplasias malignas e cirrose hepática; já nos países em desenvolvimento, a tuberculose e filariose são as causas mais comuns. Outras causas descritas são: congênita, inflamatória, pós-operatória, traumática e miscelânea. Ascite quilosa pós-operatória e pós-traumática pode ocorrer precocemente (ao redor de 1 semana) por lesão de vasos linfáticos, ou tardiamente (semanas a meses), devido a aderências ou compressão extrínseca de vasos linfáticos ${ }^{3}$. As operações que podem resultar em ascite quilosa incluem correção de aneurisma de aorta abdominal, dissecção de linfonodos retroperitoneais, fundoplicatura laparoscópica a Nissen ${ }^{4}$, implante de cateter para diálise peritoneal, derivações esplenorrenais distais, nefrectomia laparoscó- 
pica em doador renal e transplante hepático. Ascite quilosa pós-operatória geralmente resulta de uma fistula entre a cisterna do quilo ou linfáticos intestinais e a cavidade peritoneal, representando uma complicação significativa com sérias implicações metabólicas, nutricionais e imunológicas.

O quilotórax, presente nos casos apresentados, tem causa traumática em $50 \%$ dos casos, podendo ser causado por lesão do ducto torácico acima do diafragma ou por lesão do ducto em sua porção intra-abdominal com passagem da secreção quilosa para o interior da cavidade pleural. Os procedimentos cirúrgicos mais relacionados são esofagectomia e cirurgia para cardiopatia congênita, porém podem acontecer após vários procedimentos abdominais e cervicais ${ }^{5}$, o que dificulta a definição de qual porção do ducto está lesada, como ocorreram nos casos descritos.

As manifestações clínicas podem ser perda de peso, dispneia, dor abdominal inespecífica, desnutrição, náuseas, saciedade precoce, febre, sudorese noturna ${ }^{2,6}$. A paracentese é a principal ferramenta diagnóstica, o líquido apresenta-se tipicamente esbranquiçado, com níveis de triglicerídeos acima de $110 \mathrm{mg} / \mathrm{dL}^{1,7}$. Ecografia abdominal e tomografia computadorizada de abdome auxiliam no diagnóstico ao mostrar coleção líquida no abdome e orientar a paracentese. A linfocintilografia é útil para identificação do local de drenagem da linfa e pode orientar a intervenção cirúrgica ${ }^{8}$.

O tratamento clínico consiste em dieta com alto teor de proteína e baixo de gordura, nutrição parenteral total e administração de somatostatina ou octreotide. A restrição dietética de triglicerídeos de cadeia longa evita sua conversão em monoglicerídeos e ácidos graxos livres, que são transportados como quilomicrons para os ductos linfáticos intestinais. Dessa forma uma dieta com pouca gordura e suplementação de triglicerídeos de cadeia média reduz a produção e fluxo do quilo. A nutrição parenteral total é o próximo passo no tratamento, já que rapidamente reduz o fluxo de linfa e apresenta taxa de sucesso maior que $50 \% \%^{9,10}$. A somatostatina e octreotide agem através da inibição da secreção linfática através de receptores específicos localizados na parede dos vasos linfáticos intestinais ${ }^{11-13}$. Em pacientes com ascite volumosa, paracentese de alívio deve ser realizada e repetida quando necessário para aliviar o desconforto abdominal e dispneia.
O tratamento cirúrgico tem mostrado benefícios em casos de ascite quilosa pós-operatória, neoplásica ou congênita, sendo recomendada a linfografia ou linfocintilografia antes do procedimento como tentativa de identificação do local anatômico de vazamento. Porém, devido à dificuldade em acessar o local da fístula e pelas aderências encontradas na reoperação, o sucesso cirúrgico é limitado. Algumas variações anatômicas também podem dificultar a abordagem cirúrgica: o ducto torácico pode ter início baixo (L1-L2), sendo acessível pela secção do pilar. É frequente, todavia, seu início em T11-T12, o que impede o acesso abdominal, podendo levar ao insucesso do tratamento. $\mathrm{O}$ acesso torácico é mais utilizado para tratar refluxo primário ou obstruções altas, normalmente tumorais, podendo ser realizado para fins de ligadura nos casos de ascite quilosa. A identificação do local de extravasamento durante o procedimento cirúrgico pode ser facilitada pela ingestão no pré-operatório de alimentos gordurosos ou no pré-operatório de óleo mineral ou azul patente por sonda nasogástrica, porém nem sempre esse objetivo é obtido.

Jensen \& Weiss ${ }^{14}$ descreveram um caso de ascite quilosa após colecistectomia videolaparoscópica, tendo utilizado ligadura e cola de fibrina para fechar o ponto de vazamento no leito hepático da vesícula biliar.

Existem vários casos descritos de ascite quilosa após correção de aneurisma de aorta abdominal, com taxa de mortalidade de 11 a 18\%. Leibovich et al. ${ }^{3}$, em uma revisão de 102 casos de ascite quilosa, encontrou $81 \%$ deles associados a cirurgia em aorta abdominal. Busch et al. ${ }^{15}$ realizaram múltiplas ligaduras de vasos linfáticos próximos à anastomose entre a aorta e a prótese seguidos de omentoplastia. A paciente persistiu, porém, com drenagem de secreção quilosa, e somente após nutrição parenteral total e controle dietético por 2 meses houve cura da ascite quilosa. O shunt peritoneovenoso foi descrito por LeVeen et al. ${ }^{16}$, que trataram com sucesso 9 de 10 casos de ascite quilosa, procedimento repetido por Sarazin \& Sauter ${ }^{17}$ e Fleisher et al. ${ }^{18}$ no tratamento de ascite quilosa após correção de aneurisma de aorta abdominal. No entanto, Press \& Kaufman ${ }^{19}$ realizaram esse procedimento em quatro pacientes, e em todos os casos houve oclusão da derivação. Esse procedimento não é mais utilizado atualmente devido a alta taxa de complicações como sepse coagulação intravascular disseminada, distúrbios eletrolíticos, embolia aé- 
rea, oclusão intestinal e oclusão do próprio shunt, devido a alta viscosidade da linfa ${ }^{20,21}$.

A videolaparoscopia tem mostrado avanço no tratamento da ascite quilosa, por confirmar o diagnóstico, realizar a drenagem da ascite, e para a procura e tratamento do local de drenagem quilosa no abdome ou retroperitônio. $\mathrm{O}$ tratamento pode ser feito através de ressecção de tecidos linfodisplásicos, ligadura de vasos linfáticos incompetentes, tratamento com laser de dióxido de carbono dos vasos linfáticos dilatados ou shunts microcirúrgicos linfoveno$\operatorname{sos}^{19-23}$. No segundo caso apresentado, a esclerose dos vasos linfáticos junto aos pilares diafragmáticos realizada por via videolaparoscópica obteve sucesso.

O quiloperitôneo pós-traumático é uma lesão rara, com aproximadamente 16 casos descritos após trauma contuso, mais da metade em crianças ${ }^{23,24}$. Existem cinco casos descritos de lesão isolada do ducto biliar. Geralmente, a lesão do ducto biliar é acompanhada de lesão de outros órgãos, como o fígado, duodeno, rim e pâncreas. A apresentação clínica do quiloperitôneo geralmente é inespecífica, mas a tomografia computadorizada de abdome pode mostrar uma coleção líquida com nível gorduralíquido, patognomônica da condição. A abordagem imediata da lesão traumática deve ser a simples ligadura do ducto linfático, tendo sido descrita a utilização da cola de fibrina como selante ${ }^{25}$. Não há evidência da necessidade de medidas adicionais no pós-operatório como nutrição parenteral ou octreotide ${ }^{23}$. O manejo da ascite quilosa traumática em fase crônica segue os mesmos princípios da ascite quilosa pós-operatória.

O primeiro caso apresentado refere-se a ascite quilosa pós-traumática, entidade clínica de difícil diagnóstico, investigação e manejo, necessitando de vários recursos diagnósticos e terapêuticos para elucidação e resolução do caso. A ligadura dos vasos linfáticos junto aos pilares diafragmáticos é de difícil realização técnica e nem sempre traz os resultados esperados com o obtido nos casos relatados.

\section{Conclusão}

O manejo da ascite quilosa ainda é um desafio terapêutico nos dias atuais, trazendo alta morbidade aos pacientes e desafio para aqueles que os tratam. O tratamento inicial deve consistir em medidas dietéticas e nutrição parenteral, deixando-se o tratamento cirúrgico para os casos refratários. A ligadura de vasos linfáticos por via aberta ou videolaparoscópica faz parte das opções cirúrgicas e pode ser efetiva em casos selecionados.

\section{Referências}

1. Cárdenas A, Chopra S. Chylous ascites. Am J Gastroenterol. 2002;97:1896-9000.

2. Browse NL, Wilson NM, Russo F, Al-Hassan H, Allen DR. Aetiology and treatment of chylous ascites. Br J Surg. 1992;79:1145-50.

3. Leibovich I, Mor Y, Golomb J, Ramon J. The diagnosis and management of postoperative chylous ascites. J Urol. 2002;167:449-57.

4. Bacelar TS, de Albuquerque AC, de Arruda PC, Ferraz AA, Ferraz EM. Postoperative chylous ascites: a rare complication of laparoscopic Nissen fundoplication. JSLS. 2003;7:269-71.

5. Doerr CH, Allen MS, Nichols FC 3rd, Ryu JH. Etiology of chylothorax in 203 patients. Mayo Clin Proc. 2005;80:86770.

6. Aalami OO, Allen DB, Organ CH Jr. Chylous ascites: a collective review. Surgery. 2000;128:761-78.

7. Runyon BA, Hoefs JC, Morgan TR. Ascitic fluid analysis in malignancy-related ascites. Hepatology. 1988;8:1104-9.

8. Pui MH, Yueh TC. Lymphoscintigraphy in chyluria, chyloperitoneum and chylothorax. J Nuc Med. 1998;39:1292-6.

9. Lee YY, Soong WJ, Lee YS, Hwang B. Total parenteral nutrition as a primary therapeutic modality for congenital chylous ascites: report of one case. Acta Paediatr Taiwan. 2002;43:214-6.

10. Alliët P, Young C, Lebenthal E. Chylous ascites: total parenteral nutrition as primary therapeutic modality. Eur J Pediatr. 1992;151:213-4.

11. Leong, RW, House, AK, Jeffrey, GP. Chylous ascites caused by portal vein thrombosis treated with octreotide. J Gastroenterol Hepatol. 2003;18:1211

12. Mincher L, Evans J, Jenner MW, Varney VA. The successful treatment of chylous effusions in malignant disease with octreotide. Clin Oncol (R Coll Radiol). 2005;17:118-21.

13. Huang Q, Jiang ZW, Jiang J, Li N, Li JS. Chylous ascites: treated with total parenteral nutrition and somatostatin. World J Gastroenterol. 2004;10:2588-91.

14. Jensen EH, Weiss CA 3rd. Management of chylous ascites after laparoscopic cholecystectomy using minimally invasive techniques: a case report and literature review. Am Surg. 2006;72:60-3.

15. Busch T, Lotfi S, Sirbu H, Aleksic I, Dalichau H. Chyloperitoneum. A rare complication after abdominal aortic aneurysm repair. J Cardiovasc Surg. 2000;41:617-8.

16. LeVeen HH, Wapnick S, Grosberg S, Kinney MJ. Further experience with peritoneo-venous shunt for ascites. Ann Surg. 1976;184:574-81.

17. Sarazin WG, Sauter KE. Chylous ascites following resection of a ruptured abdominal aneurysm. Treatment with a peritoneovenous shunt. Arch Surg. 1986;121:246-7.

18. Fleisher HL 3rd, Oren JW, Sumner DS. Chylous ascites after abdominal aortic aneurysmectomy: successful management with a peritoneovenous shunt. J Vasc Surg.1987;6:403-7. 
19. Press OW, Press NO, Kaufman SD. Evaluation and management of chylous ascites. Ann Intern Med. 1982;96:358-64.

20. Ablan CJ, Littooy FN, Freeark RJ. Postoperative chylous ascites: diagnosis and treatment. A series report and literature review. Arch Surg. 1990;125:270-3.

21. Voros D, Hadziyannis S. Successful management of postoperative chylous ascites with a peritoneojugular shunt. J Hepatol. 1995;22:380.

22. Skala J, Witte C, Bruna J, Case T, Finley P. Chyle leakage after blunt trauma. Lymphology. 1992;25:62-8.

23. Meinke AH 3rd, Estes NC, Ernst CB. Chylous ascites following abdominal aortic aneurysmectomy. Management with total parenteral hyperalimentation. Ann Surg. 1979;190:631-3.
24. Beal AL, Gormley CM, Gordon DL, Ellis CM. Chylous ascites: a manifestation of blunt abdominal trauma in an infant. J Pediatr Surg. 1998;33:650-2.

25. Vollman R, Keenan W, Eraklis A. Post traumatic chylous ascites in infancy. N Eng J Med. 1966;275:875-7.

Correspondência:

Claudia Stein Gomes

Rua Padre Anchieta 2004/1302

CEP 80730-000 - Curitiba, PR

Tel.: (41) 3320.3526

E-mail: steingomes@hotmail.com 